\title{
In Pursuit of Adult Progenitors of Thymic Epithelial Cells
}

\author{
Tatsuya Ishikawa ${ }^{1,2}$, Nobuko Akiyama $^{3}$ and Taishin Akiyama ${ }^{1,2 *}$ \\ ${ }^{1}$ Laboratory of Immune Homeostasis, RIKEN Center for Integrative Medical Sciences, Yokohama, Japan, ${ }^{2}$ Graduate School \\ of Medical Life Science, Yokohama City University, Yokohama, Japan, ${ }^{3}$ Laboratory for Immunogenetics, RIKEN Center of \\ Integrative Medical Sciences, Yokohama, Japan
}

Peripheral T cells capable of discriminating between self and non-self antigens are major components of a robust adaptive immune system. The development of self-tolerant $T$ cells is orchestrated by thymic epithelial cells (TECs), which are localized in the thymic cortex (cortical TECs, cTECs) and medulla (medullary TECs, mTECs). cTECs and mTECs are essential for differentiation, proliferation, and positive and negative selection of thymocytes. Recent advances in single-cell RNA-sequencing technology have revealed a previously unknown degree of TEC heterogeneity, but we still lack a clear picture of the identity of TEC progenitors in the adult thymus. In this review, we describe both earlier and recent findings that shed light on features of these elusive adult progenitors in the context of tissue homeostasis, as well as recovery from stress-induced thymic atrophy.

\section{OPEN ACCESS}

Edited by:

Izumi Ohigashi,

Tokushima University, Japan

Reviewed by:

Clare C. Blackburn,

University of Edinburgh,

United Kingdom

Maude Dumont-Lagacé,

Montreal University, Canada

*Correspondence:

Taishin Akiyama

taishin.akiyama@riken.jp

Specialty section:

This article was submitted to

T Cell Biology,

a section of the journal

Frontiers in Immunology

Received: 27 October 2020 Accepted: 08 February 2021 Published: 25 February 2021

Citation:

Ishikawa T, Akiyama N and Akiyama T (2021) In Pursuit of Adult Progenitors of Thymic Epithelial Cells.

Front. Immunol. 12:621824.

doi: 10.3389/fimmu.2021.621824
Keywords: thymus, thymic epithelial cells, immunodeficiency, immune dysregulation, single cell RNA-sequencing

\section{INTRODUCTION}

Once regarded as a vestigial organ that had lost its function during evolution, the thymus is now recognized as a primary lymphoid organ that performs irreplaceable functions in differentiation and selection of self-tolerant $\mathrm{T}$ cells (1). The thymus "educates" $\mathrm{T}$ cells by allowing those that possess a T-cell-receptor capable of interacting with major histocompatibility complex class I or II (MHCI and MHCII) molecules to survive, while eliminating those that recognize self-antigens presented by MHCI or MHCII molecules. This event is orchestrated by two types of thymic epithelial cells (TECs) that reside in the thymic cortex (cortical thymic epithelial cells, cTECs) and medulla (medullary thymic epithelial cells, mTECs). Functionally, cTECs are mainly required for lineage commitment, expansion, and positive selection of thymocytes, while mTECs promote negative selection of self-reactive $\mathrm{T}$ cells or promote their diversification into the regulatory $\mathrm{T}$ cell lineage by ectopically expressing self-derived tissue-specific antigens (2-4). The expression of tissue-specific antigens is partially regulated by the autoimmune regulator (AIRE), the mutation and dysfunction of which contribute to severe autoimmune diseases (5).

In the past several years, intense effort has focused on understanding the development of TECs, and bipotent and unipotent progenitors of cTECs and mTECs have been rigorously studied. The presence of embryonic bipotent progenitors of cTECs and mTECs was evidenced by transplanting a single early embryonic TEC (day 12.5) into the fetal thymus to generate both cTECs and mTECs (6), and by neonatal reactivation of developmentally arrested fetal bipotent progenitors (7). These embryonic bipotent progenitors can be identified by expression of placenta-expressed transcript 1 (PLET1), and resemble the phenotype of cTECs expressing CD205, $\beta 5$ t, and IL-7 (8-12). Although evidence regarding embryonic unipotent progenitors of cTECs is limited (13), equivalent progenitors of mTECs have been well-studied (14-16). Overall, it is most likely that mTEC progenitors and cTECs are derived from bipotent progenitors in the embryonic thymus, although mechanisms underlying fate decisions of bipotent TEC progenitors remain elusive. 
In contrast to embryonic TEC progenitors, little is known about the corresponding progenitors that maintain thymic tissue function in adults. In this review, we first highlight some reported properties of adult progenitors under homeostatic conditions, and then review how the putative adult progenitors contribute to thymic regeneration, from both the cellular and molecular perspectives, following stress-induced damage to the thymus.

\section{ADULT TEC PROGENITORS UNDER HOMEOSTATIC CONDITIONS}

In vivo cell labeling and ablation studies suggested that TECs are able to undergo turnover in the adult thymus $(14,17,18)$. Adult progenitors or stem cells of TECs should exist to maintain the steady-state functions of the thymus so as to repopulate the periphery with immunologically competent $\mathrm{T}$ cells. In this section, we describe some recent findings on the identity of these elusive adult TEC progenitors under steady-state conditions.

\section{Differences Between Embryonic and Adult TEC Progenitors}

Earlier studies have suggested that some molecular features of TEC progenitors may differ between embryonic and adult thymus. Although mTEC-restricted progenitors are enriched in cells expressing the embryonic stem cell marker, SSEA-1, within the CLAUDIN-3/4-positive (CLD3/4 ${ }^{\text {hi }}$ ) population of the fetal thymus (16), these fractions appear to lose their self-replicating capacity in the adult thymus (16). Most SSEA- $1^{+}$CLD3/4 ${ }^{\text {hi }}$ TECs in the adult thymus are $\mathrm{MHCII}^{\mathrm{lo} /-}$ cells and express keratin 10 , a marker of terminally differentiated mTECs known as PostAIRE mTECs $(16,19-22)$. Moreover, unlike $\beta 5 t^{+}$embryonic bipotent progenitors, adult $\beta 5 \mathrm{t}^{+}$cells contribute minimally to maintenance of TECs $(23,24)$. In light of these findings, Ohigashi et al. (23) argued that although adult progenitors are derived from embryonic $\beta 5 t^{+}$bipotent progenitors, they develop into mTEC-restricted SSEA- ${ }^{+}$CLD3/4 ${ }^{\text {hi }}$ progenitors after losing $\beta 5$ t expression.

\section{Adult Bipotent Progenitors}

The existence of an adult bipotent progenitor capable of supplying cTECs and mTECs was tested using the thymic reaggregation/transplantation approach $(25,26)$. Ulyanchenko et al. demonstrated that TECs expressing LY51 (a marker of cTECs) and PLET1 had progenitor activities. Notably, the activity of bipotent progenitors generating both cTECs and mTECs were present in the $\mathrm{MHCII}^{+}$fraction of $\mathrm{Ly}_{51}{ }^{+} \mathrm{PLET}_{1}{ }^{+}$TECs (hereafter referred to as PLET1 ${ }^{+}$TECs) (25). On the other hand, Wong et al. (26) proposed that bipotent progenitors are present in a subset of TECs expressing low levels of MHCII and LY51 and lacking the mTEC marker UEA-1 ligand (referred to as TEC ${ }^{\text {lo }}$ ). They both found that bipotent progenitors were present in UEA1-negative TEC fractions, and that they express surface LY51 and Pax1 mRNA, suggesting their similarity to cTECs. Unfortunately, there are some discrepancies between these studies. For example, PLET $^{+}$TECs are enriched in the MHCII ${ }^{\text {hi }}$ fraction, but TEC ${ }^{\text {lo }}$ belongs to $\mathrm{MHCII}^{\mathrm{lo}}$ fraction $(25,26)$. $\mathrm{PLET}^{+}{ }^{+}$TECs comprise
$<1 \%$ of all TECs, and limiting dilution analysis suggested their bipotency at nearly clonal resolution (25). In contrast, TEC ${ }^{\text {lo }}$ comprises about $20 \%$ of all TECs (26). Therefore, it may be possible that both unipotent CTEC and mTEC progenitors could be present in $\operatorname{TEC}^{\text {lo }}(25,26)$. Importantly, as both studies verified their differentiation potential using reaggregation with fetal thymic cells, such conditions may not be suitable to address adult progenitors. Moreover, details of experimental conditions for thymic reaggregation differed slightly between two studies, which may explain the discrepancy. In vivo fate mapping needs to be performed in the adult thymus to evaluate their physiological fate.

\section{A Subset of mTEC ${ }^{l o}$ Cells Represents mTEC Lineage-Restricted Adult Progenitors}

Previously, mTECs were categorized as mTEC $^{\text {lo }}$ or mTEChi, depending on expression levels of AIRE, CD80, and MHCII. mTEC $^{\text {lo }}$ cells expressing lower levels of AIRE, CD80, and MHCII have been considered as an immature stage of mTEC $^{\text {hi }}(17,27-$ 29). However, recent findings demonstrated that the mTEC $^{\text {lo }}$ fraction contains multiple subsets. Several studies showed that $\mathrm{AIRE}^{+}$mTEC $^{\text {hi }}$ can further differentiate into mTECs with lower expression of AIRE, CD80, and MHCII (Post-AIRE mTECs), which are included in the mTEC $^{\text {lo }}$ fraction (19-22). Moreover, Lucas et al. (30) demonstrated that the mTEC $^{\text {lo }}$ fraction can be segregated by expression of the chemokine, CCL21, into CD $104^{+}{ }^{-} \mathrm{CCL} 21^{+}$and CD $104^{-} \mathrm{CCL}_{2} 1^{-}$subsets. Since CCL21 recruits positively selected thymocytes to the thymic medulla, the $\mathrm{CD} 104^{+} \mathrm{CCL}_{2} 1^{+}$mTEC $^{\text {lo }}$ subset may be considered functionally mature cells (31). Onder et al. (32) reported that within CD80TECs, there is a population of podoplanin (Pdpn)-expressing mTEC-restricted progenitors localized in the cortical-medullary junction (junctional TECs). In summary, it is likely that a limited population of mTEC $^{\text {lo }}$ cells should be unipotent progenitors of $\mathrm{mTEC}^{\text {hi }}$.

\section{New Insights Gained From Single-Cell RNA-Sequencing Studies}

Recent progress in single-cell RNA-sequencing (scRNA-seq) technology has uncovered a previously unknown degree of heterogeneity among TECs and has provided new insights into both the developmental pathway and mechanism of TECs, especially mTECs under homeostatic conditions (33-36). Based on the cell type clusters obtained from scRNA-seq results, Bornstein et al. categorized mTECs into four subsets: mTEC I, mTEC II, mTEC III, and a newly identified mTEC IV or tuft cells with chemosensory properties $(33,35)$. With respect to previous mTEC classifications, the MTEC I, MTEC II, and mTEC III subsets are equivalent to CCL $21^{+}$mTEC $^{\text {lo }}$, $\mathrm{AIRE}^{+}$ mTEC $^{\text {hi }}$, and Post-AIRE mTEC subsets, respectively. Notably, Lucas et al. (30) showed that DCLK1 ${ }^{+}$mTEC IV/tuft cells are enriched in the $\mathrm{CD} 104^{-} \mathrm{CCL}_{2} 1^{-} \mathrm{mTEC}^{\mathrm{lo}}$ subset. Additionally, Dhalla et al. (34) used scRNA-seq to more deeply interrogate mTEC heterogeneity. These authors identified a "Proliferating mTEC" cluster that seemed to bridge the clusters representing 
mature $\mathrm{AIRE}^{+}$mTEC $^{\text {hi }}$ and CCL2 $1^{+}$mTEC $^{\text {lo }}$ (34). Cells in the "Proliferating mTEC" cluster exhibited upregulation of genes involved in proliferation, such as Mki67, and expressed Aire, suggesting that it could represent proliferating mTECs previously reported within mTEC $^{\text {hi }}(14,26,34)$. The trajectory of diffusion pseudotime analysis suggested that cells in the "Proliferating mTEC" cluster could act as bipotent MTEC progenitors that differentiate into both $\mathrm{AIRE}^{+}$mTEC $^{\text {hi }}$ and CCL21 ${ }^{+}$mTEC $^{\text {lo }}$ lineages (34). However, analysis of the same data using RNA velocity, a different trajectory method that relies on pre- and post-spliced RNA reads (37), produced conflicting results. The latter analysis indicated that rather than differentiating into

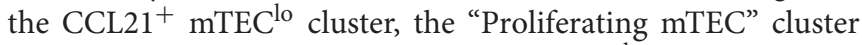
seemed to be derived from CCL $21^{+}$mTEC $^{\text {lo }}$ and junctional TEC clusters (34). In a different study, Baran-Gale et al. (38) conducted scRNA-seq of mouse TECs throughout the 1st year of life and studied their various trajectories using genetic fate mapping under control of the $\beta 5 \mathrm{t}$ promoter. They also identified a cluster equivalent to the "Proliferating mTECs" (38). However, their diffusion pseudotime analysis failed to suggest that the "Proliferating mTEC" cluster was positioned at the branch point between mTEC differentiation into $\mathrm{AIRE}^{+}$mTEC ${ }^{\text {hi }}$ and CCL21 ${ }^{+}$ $\mathrm{mTEC}^{\text {lo }}$ lineages (38). Instead, they showed that the "Intertypical TEC" cluster, which encompassed cTEC, CCL $21^{+}$mTEC $^{\text {lo }}$, and junctional TEC, could bifurcate into two mTEC trajectories that both progressed toward $\mathrm{AIRE}^{+}$mTEC hi via the "Proliferating mTEC" cluster (38). In a more recent study, Wells et al. (39) showed that the "TAC-TEC" cluster, a cluster equivalent to the "Proliferating mTECs," could give rise to both $\mathrm{AIRE}^{+}$ mTEC $^{\text {hi }}$ and CCL2 $1^{+}$mTEC $^{\text {lo }}$ lineages, using RNA velocity. In summary, in silico predictions of mTEC differentiation dynamics deduced from scRNA-seq data were split. These discrepancies could be due to differences in cell coverages and sequencing depths detected using different scRNA-seq methods (Table 1). For instance, low numbers of detected mRNA species could affect RNA velocity analysis, which relies on detection of unspliced mRNAs occupying $15-25 \%$ of total sequencing reads in scRNA data (37).

Notably, the "Intertypical TEC" cluster contained PLET1 ${ }^{+}$ TECs and expressed markers associated with the bipotent TEC $^{\text {lo }}$ progenitors, which were identified by cytometry-based analysis as described above $(25,26,38)$. Diffusion pseudotime analysis showed that the "Intertypical TEC" cluster could not only contribute to the MTEC lineage, but also to the cTEC lineage, suggesting its bipotency (38). Additionally, these authors suggested that the aging "Intertypical TEC" cluster displayed features of progressive quiescence, and that it could arise from either $\beta 5 \mathrm{t}^{+}$or $\beta 5 \mathrm{t}^{-}$progenitors independently (38). This proposal contradicts the argument put forth by Ohigashi et al. (23) that $\beta 5 t^{+}$and $\beta 5 t^{-}$progenitors possess a precursorproduct relationship.

Importantly, all of the clusters described above, which are identified in mouse analyses, can also be identified in scRNAseq data obtained from the human thymus, indicating that the cluster-based classification is not restricted to mice (38, 40). Nevertheless, we await experimental verification of the existence and function of putative adult progenitors inferred from computationally defined clusters.

\section{ADULT TEC PROGENITORS DURING RECOVERY FROM STRESS-INDUCED DAMAGE}

Adult progenitors would be integral not only to maintaining tissue homeostasis, but also to recovery of the thymus from stress-induced damage. In the following sections, we describe how putative adult TEC progenitors could contribute to thymic recovery at both the cellular and molecular levels, based on studies using mouse models that mimic insults.

\section{Repair Potential of the Damage-Sensitive Thymus}

The thymus is extremely sensitive to damage and exposure to acute or chronic insults results in a pronounced decline in cellularity, a phenomenon known as thymic atrophy (41, 42). For example, we recently demonstrated thymic atrophy displayed by mice under microgravity $(0 \mathrm{~g})$ conditions during spaceflight, which was partially mitigated by exposure to $1 \mathrm{~g}$ during spaceflight (43). After resolution of acute insults such as infections, cytoreductive therapies, and emotional and physical discomfort, the thymus is able to regenerate, although its capacity declines with age $(41,42)$. To study this endogenous thymic regeneration, researchers have employed viral and bacterial infections, sub-lethal irradiation, and synthetic corticosteroid injections to model acute insults (44-50). Chronic insults, such as aging, hamper the ability of the thymus to regenerate, but age-induced defects in recovery can be transiently reversed by ablation of sex steroids (51-53). This can be explained by the ability of sex hormones to induce apoptosis and to inhibit proliferation of developing $\mathrm{T}$ cells $(54,55)$. In fact, the

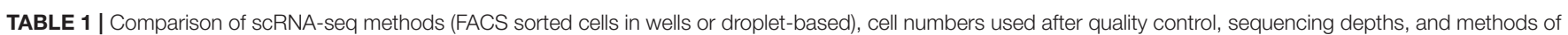
trajectory analysis used by different scRNA-seq studies from postnatal thymus glands of wild-type mice under homeostasis.

\begin{tabular}{|c|c|c|c|c|}
\hline & scRNA-seq method & Number of cells used in analysis & Sequencing depth per cell & Trajectory analysis \\
\hline Bornstein et al. (33) & MARS-seq (FACS) & $1,825 \mathrm{CD} 45^{-}$cells and 1,716 TECs & Median of 1,711 UMls & None \\
\hline Dhalla et al. (34) & 10X Genomics (Droplet) & 6,894 mTECs & Median of 1,830 genes & Diffusion pseudotime RNA velocity \\
\hline Baren-Gale et al. (38) & SMART-Seq2 (FACS) & 2,327 TECs & Not mentioned & Diffusion pseudotime \\
\hline Wells et al. (39) & 10X Genomics (Droplet) & 2,434 TECs & 200-7,500 genes & RNA velocity \\
\hline
\end{tabular}




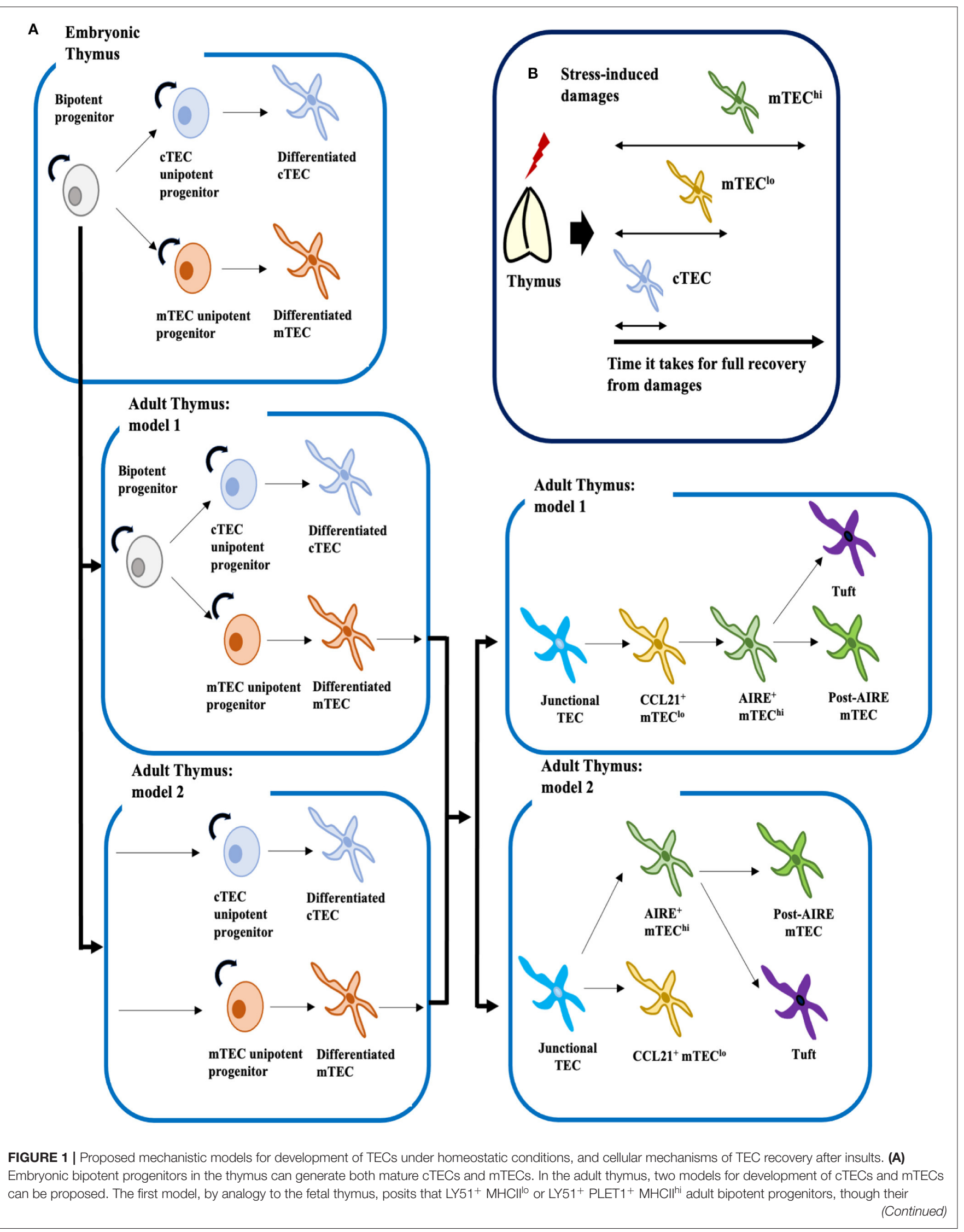


FIGURE 1 | identities remain controversial, supply both mature cTECs and mTECs. The second model suggests that adult bipotent progenitors are lost with age, and that unipotent progenitors committed to either the cTEC or mTEC lineages supply mature cTECs and mTECs. However, these two models are not necessary mutually exclusive. Similarly, two models can be proposed for development of mTEClo and mTEChi: one supports linear differentiation from junctional TECs to mature mTEC hi via CCL21+ $\mathrm{mTEC}^{\mathrm{lo}}$, and the other suggests bifurcation of junctional TECs into mature CCL21+ $\mathrm{mTEC}^{\mathrm{lo}}$ and $\mathrm{AIRE}^{+} \mathrm{mTEC}{ }^{\text {hi }}$. Mature AIRE ${ }^{+} \mathrm{mTEC}{ }^{\text {hi }}$ would then differentiate into Post-AIRE mTECs or tuft cells $(4,35) \mathbf{( B )}$ Mouse models of thymic stress have shown that cTECs are the first cell type to recover from insults, followed by mTEC ${ }^{\circ}$ and then mTEC ${ }^{\text {i. }}$ Therefore, it is possible that the adult progenitor population may be confined to cTECs.

regenerative capacity of the thymus was known well before its function as a lymphoid organ was discovered $(56,57)$.

\section{Cellular Mechanisms of TEC Regeneration}

Several studies have shed light on cellular mechanisms of TEC regeneration, which appear to initiate from the putative adult progenitors. Using an irradiation-induced stress model, we recently performed a quantitative analysis of TEC regeneration and its mathematical modeling (58). We showed that full recovery was reached earlier by cTECs than mTECs, and that mTEC recovery might be negatively regulated by $\mathrm{CD} 4^{+} \mathrm{CD} 8^{+}$ double-positive $\mathrm{T}$ cells (58). Similar results were obtained by Dudakov et al. (59), suggesting that cTECs and mTEC ${ }^{\text {lo }}$ subsets were the major contributors to TEC recovery. Dumont-Lagacé et al. (60) used the tetracycline-inducible H2B-GFP mouse model to tag slow-cycling label-retaining cells (LRCs), which hypothetically label quiescent stem cells in the adult thymus. Following induction of acute injury by exposure of mice to irradiation, $\mathrm{UEA}^{-}$LRCs displayed a significant increase in proliferation (60). Interestingly, these LRCs were localized in the cortical-medullary junction and were proposed as adult progenitors (60). Recently, Lepletier et al. (61) used the aging/sex steroid-ablation model to demonstrate that during recovery, there was a decrease in the ratio of $\mathrm{MHCII}^{\mathrm{lo}}$ cTEC to MHCII ${ }^{\text {hi }}$ cTEC, followed by a decrease in the ratio of mTEC $^{\text {lo }}$ to mTEC $^{\text {hi }}$. Taken together, these studies consistently showed that cTECs sharing similar phenotypes to the putative adult bipotent progenitors of TECs can initiate thymic recovery from stress, and potentially could contain a subpopulation of adult progenitors $(25,26)$.

\section{Molecular Mechanisms of TEC Regeneration}

Many soluble factors are involved in endogenous regeneration of TECs after damage. Following sublethal irradiation, depletion of radiosensitive $\mathrm{CD} 4^{+} \mathrm{CD}^{+}$double-positive $\mathrm{T}$ cells triggers radioresistant innate lymphoid cells to produce IL-22 $(59,62,63)$. IL-22 binding to the IL-22 receptor on TECs then promotes TEC recovery through phosphorylation of STAT3 and STAT5 $(59,63)$. Of note, cTECs and mTEC $^{\text {lo }}$, but not mTEC $^{\text {hi }}$ subsets showed significant early responses to administration of IL-22 during recovery (59).

An alternative mechanism of recovery involves keratinocyte growth factors (KGF). KGF is mainly expressed by fibroblasts and its cognate receptor, FgfR2IIIb, is exclusively expressed by TECs in the thymus (64-67). Importantly, administration of KGF to mice exposed to irradiation accelerated the recovery of TECs by enhancing their proliferation (67).
Bone morphogenetic protein 4 (BMP4) also participates in mediating thymic regeneration. BMP4 is predominantly expressed by fibroblasts and radioresistant endothelial cells, and its expression increases soon after radiation exposure (68). Remarkably, administration of thymus-derived ex vivopropagated endothelial cells, but not of endothelial cells derived from other organs, rescued the damage to TECs in mice exposed to radiation (68). Such rescue was driven by increased proliferation of cTECs, reflecting their higher expression of the non-redundant receptor, BMPR2, compared with mTECs (68).

\section{CONCLUSIONS AND FUTURE PERSPECTIVES}

Despite striking progress owing to advances in scRNA-seq technology, the exact mechanisms by which TECs develop remain far from clear, especially in postnatal and adult thymus. Based on our current understanding, we can propose two potential models for the development of cTECs and mTECs (Figure 1A). Given that early progenitors in the adult could arise from $\beta 5 \mathrm{t}^{+}$embryonic bipotent progenitors, mature cTECs and mTECs could be replenished by bipotent progenitors or/and by two types of unipotent progenitors separately committed to the cTEC or mTEC lineages $(8,9,11,23)$. Studies of TEC development under homeostatic and recovery conditions suggest that these early progenitors bear cTEC phenotypes (Figure 1B) $(25,26,58-61)$.

Recent findings also suggest that similar to mTEC $^{\text {hi }}$, mTEC $^{\text {lo }}$ could also contain functionally mature cells $(30,31,34)$. Thus, two models can be proposed for development of the mTEC lineage: one supports bifurcation from junctional TECs into terminally differentiated $\mathrm{mTEC}^{\mathrm{hi}}$ and CCL $21^{+} \mathrm{mTEC}^{\mathrm{lo}}$, and the other, which is grounded in the traditional view that mTEC $^{\text {lo }}$ are immature, supports the linear differentiation pathway from junctional TECs to mature mTEC $^{\text {hi }}$ via CCL2 $1^{+}$mTEC $^{\text {lo }}$ (Figure 1A). Further experimentation will be necessary to verify developmental trajectories proposed from in silico analyses. Notably, cell type clusters identified in scRNA-seq analyses by Baran-Gale et al. (38) contained mixtures of cells from TEC subsets defined by flow cytometry. Hence, new cell markers will be needed to equate cytometrically defined cell types with the computationally derived cell clusters.

Another key point is the possibility that progenitors identified under homeostatic conditions could differ from those present during TEC recovery. Interestingly, whereas IL-22 is critical for TEC recovery from radiation-inducing damage, deletion of IL-22 did not significantly affect TEC cellularity in steady state conditions. This implies that the TEC progenitor potential 
may depend on thymic microenvironments. Moreover, the discrepancy in the two studies reporting bipotent adult TEC progenitors may be explained by possible variations and plasticity of TEC progenitors. Indeed, in other epithelial tissues such as the small intestine, skin, mammary gland, and lung, the potential of stem cells appears to change depending on the microenvironment (e.g., niches) and conditions in tissues (69). Cells that lack stemness during steady-state conditions have the capacity to acquire features of stem cells under different settings (70). To resolve similar complexity of TEC progenitors, an in vivo fate-mapping study using a specific progenitor marker driving CRE should be useful.

Finally, identification of TEC progenitors has crucial clinical implications. For example, therapeutic targeting of these cells would provide new opportunities for reversal of thymic aging, thereby boosting the responsiveness of an individual's immune

\section{REFERENCES}

1. Miller J. The golden anniversary of the thymus. Nat Rev Immunol. (2011) 11:489-95. doi: 10.1038/nri2993

2. Abramson J, Anderson G. Thymic epithelial cells. Annu Rev Immunol. (2017) 35:85-118. doi: 10.1146/annurev-immunol-051116-052320

3. Wang HX, Pan W, Zheng L, Zhong XP, Tan L, Liang Z, et al. Thymic epithelial cells contribute to thymopoiesis and $\mathrm{T}$ cell development. Front Immunol. (2019) 10:3099. doi: 10.3389/fimmu.2019.03099

4. Kadouri N, Nevo S, Goldfarb Y, Abramson J. Thymic epithelial cell heterogeneity: TEC by TEC. Nat Rev Immunol. (2020) 20:239-53. doi: 10.1038/s41577-019-0238-0

5. Anderson MS, Venanzi ES, Klein L, Chen ZB, Berzins SP, Turley SJ, et al. Projection of an immunological self shadow within the thymus by the aire protein. Science. (2002) 298:1395-401. doi: 10.1126/science.1075958

6. Rossi SW, Jenkinson WE, Anderson G, Jenkinson EJ. Clonal analysis reveals a common progenitor for thymic cortical and medullary epithelium. Nature. (2006) 441:988-91. doi: 10.1038/nature04813

7. Bleul CC, Corbeaux T, Reuter A, Fisch P, Mönting JS, Boehm T. Formation of a functional thymus initiated by a postnatal epithelial progenitor cell. Nature. (2006) 441:992-6. doi: 10.1038/nature04850

8. Baik S, Jenkinson EJ, Lane PJL, Anderson G, Jenkinson WE. Generation of both cortical and Aire $(+)$ medullary thymic epithelial compartments from CD205(+) progenitors. Eur J Immunol. (2013) 43:589-94. doi: 10.1002/eji.201243209

9. Gill J, Malin M, Hollander GA, Boyd R. Generation of a complete thymic microenvironment by MTS24(+) thymic epithelial cells. Nat Immunol. (2002) 3:635-42. doi: 10.1038/ni812

10. Bennett AR, Farley A, Blair NF, Gordon J, Sharp L, Blackburn CC. Identification and characterization of thymic epithelial progenitor cells. Immunity. (2002) 16:803-14. doi: 10.1016/S1074-7613(02)00321-7

11. Ohigashi I, Zuklys S, Sakata M, Mayer CE, Zhanybekova S, Murata S, et al. Aire-expressing thymic medullary epithelial cells originate from beta 5texpressing progenitor cells. Proc Natl Acad Sci U S A. (2013) 110:9885-90. doi: 10.1073/pnas.1301799110

12. Ribeiro AR, Rodrigues PM, Meireles C, Di Santo JP, Alves NL. Thymocyte selection regulates the homeostasis of IL-7-expressing thymic cortical epithelial cells in vivo. J Immunol. (2013) 191:1200-9. doi: 10.4049/jimmunol.1203042

13. Meireles C, Ribeiro AR, Pinto RD, Leitão C, Rodrigues PM, Alves NL. Thymic crosstalk restrains the pool of cortical thymic epithelial cells with progenitor properties. Eur J Immunol. (2017) 47:958-69. doi: 10.1002/eji.201746922

14. Gray DH, Seach N, Ueno T, Milton MK, Liston A, Lew $\mathrm{AM}$, et al. Developmental kinetics, turnover, and stimulatory system. Ultimately, such a discovery could also contribute to the field of regenerative medicine by allowing creation of thymic organoids, which could open up a new avenue of treatment for immunological disorders.

\section{AUTHOR CONTRIBUTIONS}

TI wrote the first draft of the manuscript and NA and TA critically reviewed the manuscript. All authors contributed to the article and approved the submitted version.

\section{FUNDING}

This work was supported by Grants-in-Aid for Scientific Research from JSPS (20H03441) and CREST from JST (JPMJCR2011) to TA. capacity of thymic epithelial cells. Blood. (2006) 108:3777-85. doi: 10.1182/blood-2006-02-004531

15. Hamazaki Y, Fujita H, Kobayashi T, Choi Y, Scott HS, Matsumoto M, et al. Medullary thymic epithelial cells expressing Aire represent a unique lineage derived from cells expressing claudin. Nat Immunol. (2007) 8:304-11. doi: $10.1038 /$ ni1 1438

16. Sekai M, Hamazaki Y, Minato N. Medullary thymic epithelial stem cells maintain a functional thymus to ensure lifelong central $\mathrm{T}$ cell tolerance. Immunity. (2014) 41:753-61. doi: 10.1016/j.immuni.2014.10.011

17. Gray D, Abramson J, Benoist C, Mathis D. Proliferative arrest and rapid turnover of thymic epithelial cells expressing Aire. J Exp Med. (2007) 204:2521-8. doi: 10.1084/jem.20070795

18. Rode I, Boehm T. Regenerative capacity of adult cortical thymic epithelial cells. Proc Natl Acad Sci U S A. (2012) 109:3463-8. doi: $10.1073 /$ pnas. 1118823109

19. Yano M, Kuroda N, Han H, Meguro-Horike M, Nishikawa Y, Kiyonari H, et al. Aire controls the differentiation program of thymic epithelial cells in the medulla for the establishment of self-tolerance. J Exp Med. (2008) 205:2827-38. doi: 10.1084/jem.20080046

20. White AJ, Nakamura K, Jenkinson WE, Saini M, Sinclair C, Seddon B, et al. Lymphotoxin signals from positively selected thymocytes regulate the terminal differentiation of medullary thymic epithelial cells. J Immunol. (2010) 185:4769-76. doi: 10.4049/jimmunol.1002151

21. Wang X, Laan M, Bichele R, Kisand K, Scott HS, Peterson P. PostAire maturation of thymic medullary epithelial cells involves selective expression of keratinocyte-specific autoantigens. Front Immunol. (2012) 3:19. doi: 10.3389/fimmu.2012.00019

22. Metzger TC, Khan IS, Gardner JM, Mouchess ML, Johannes KP, Krawisz $\mathrm{AK}$, et al. Lineage tracing and cell ablation identify a post-Aireexpressing thymic epithelial cell population. Cell Rep. (2013) 5:166-79. doi: 10.1016/j.celrep.2013.08.038

23. Ohigashi I, Zuklys S, Sakata M, Mayer CE, Hamazaki Y, Minato N, et al. Adult thymic medullary epithelium is maintained and regenerated by lineagerestricted cells rather than bipotent progenitors. Cell Rep. (2015) 13:1432-43. doi: 10.1016/j.celrep.2015.10.012

24. Mayer CE, Žuklys S, Zhanybekova S, Ohigashi I, Teh HY, Sansom SN, Shikama-Dorn N, et al. Dynamic spatio-temporal contribution of single $\beta 5 \mathrm{t}+$ cortical epithelial precursors to the thymus medulla. Eur J Immunol. (2016) 46:846-56. doi: 10.1002/eji.201545995

25. Ulyanchenko S, O'Neill KE, Medley T, Farley AM, Vaidya HJ, Cook AM, et al. Identification of a bipotent epithelial progenitor population in the adult thymus. Cell Rep. (2016) 14:2819-32. doi: 10.1016/j.celrep.2016.02.080

26. Wong $\mathrm{K}$, Lister NL, Barsanti M, Lim JMC, Hammett MV, Khong $\mathrm{DM}$, et al. Multilineage potential and self-renewal define an epithelial 
progenitor cell population in the adult thymus. Cell Rep. (2014) 8:1198-209. doi: 10.1016/j.celrep.2014.07.029

27. Derbinski J, Gäbler J, Brors B, Tierling S, Jonnakuty S, Hergenhahn M, et al. Promiscuous gene expression in thymic epithelial cells is regulated at multiple levels. J Exp Med. (2005) 202:33-45. doi: 10.1084/jem.20050471

28. Gäbler J, Arnold J, Kyewski B. Promiscuous gene expression and the developmental dynamics of medullary thymic epithelial cells. Eur J Immunol. (2007) 37:3363-72. doi: 10.1002/eji.200737131

29. Rossi SW, Kim MY, Leibbrandt A, Parnell SM, Jenkinson WE, Glanville SH, et al. RANK signals from CD4(+)3(-) inducer cells regulate development of Aire-expressing epithelial cells in the thymic medulla. J Exp Med. (2007) 204:1267-72. doi: 10.1084/jem.20062497

30. Lucas B, White AJ, Cosway EJ, Parnell SM, James KD, Jones ND, et al. Diversity in medullary thymic epithelial cells controls the activity and availability of iNKT cells. Nat Commun. (2020) 11:2198. doi: 10.1038/s41467-020-16041-x

31. Kozai M, Kubo Y, Katakai T, Kondo H, Kiyonari H, Schaeuble K, et al. Essential role of CCL21 in establishment of central self-tolerance in T cells. J Exp Med. (2017) 214:1925-35. doi: 10.1084/jem.20161864

32. Onder L, Nindl V, Scandella E, Chai Q, Cheng HW, Caviezel-Firner S, et al. Alternative NF- $\mathrm{\kappa B}$ signaling regulates $\mathrm{mTEC}$ differentiation from podoplaninexpressing precursors in the cortico-medullary junction. Eur J Immunol. (2015) 45:2218-31. doi: 10.1002/eji.201545677

33. Bornstein C, Nevo S, Giladi A, Kadouri N, Pouzolles M, Gerbe F, et al. Singlecell mapping of the thymic stroma identifies IL-25-producing tuft epithelial cells. Nature. (2018) 559:622-6. doi: 10.1038/s41586-018-0346-1

34. Dhalla F, Baran-Gale J, Maio S, Chappell L, Holländer GA, Ponting CP. Biologically indeterminate yet ordered promiscuous gene expression in single medullary thymic epithelial cells. Embo J. (2020) 39:e101828. doi: $10.15252 / \mathrm{embj} .2019101828$

35. Miller CN, Proekt I, von Moltke J, Wells KL, Rajpurkar AR, Wang H, et al. Thymic tuft cells promote an IL-4-enriched medulla and shape thymocyte development. Nature. (2018) 559:627-31. doi: 10.1038/s41586-018-0345-2

36. Miragaia RJ, Zhang X, Gomes T, Svensson V, Ilicic T, Henriksson J, et al. Single-cell RNA-sequencing resolves self-antigen expression during mTEC development. Sci Rep. (2018) 8:685. doi: 10.1038/s41598-017-19100-4

37. La Manno G, Soldatov R, Zeisel A, Braun E, Hochgerner H, Petukhov $\mathrm{V}$, et al. RNA velocity of single cells. Nature. (2018) 560:494-8. doi: 10.1038/s41586-018-0414-6

38. Baran-Gale J, Morgan MD, Maio S, Dhalla F, Calvo-Asensio I, Deadman ME, et al. Ageing compromises mouse thymus function and remodels epithelial cell differentiation. Elife. (2020) 9:e56221. doi: 10.7554/eLife.56221

39. Wells KL, Miller CN, Gschwind AR, Wei W, Phipps JD, Anderson MS, et al. Combined transient ablation and single-cell RNA-sequencing reveals the development of medullary thymic epithelial cells. Elife. (2020) 9:e60188. doi: 10.7554/eLife.60188

40. Park JE, Botting RA, Domínguez Conde C, Popescu DM, Lavaert M, Kunz DJ, et al. A cell atlas of human thymic development defines $\mathrm{T}$ cell repertoire formation. Science. (2020) 367:eaay3224. doi: 10.1101/2020.01.28.911115

41. Chaudhry MS, Velardi E, Dudakov JA, van den Brink RM. Thymus: the next (re)generation. Immunol Rev. (2016) 271:56-71. doi: 10.1111/imr.12418

42. Kinsella S, Dudakov JA. When the damage is done: injury and repair in thymus function. Front Immunol. (2020) 11:1745. doi: 10.3389/fimmu.2020.01745

43. Horie K, Kato T, Kudo T, Sasanuma H, Miyauchi M, Akiyama N, et al. Impact of spaceflight on the murine thymus and mitigation by exposure to artificial gravity during spaceflight. Sci Rep. (2019) 9:19866. doi: 10.1038/s41598-019-56432-9

44. Fiume G, Scialdone A, Albano F, Rossi A, Tuccillo FM, Rea D, et al. Impairment of T cell development and acute inflammatory response in HIV-1 Tat transgenic mice. Sci Rep. (2015) 5:13864. doi: 10.1038/srep13864

45. Hick RW, Gruver AL, Ventevogel MS, Haynes BF, Sempowski GD. Leptin selectively augments thymopoiesis in leptin deficiency and lipopolysaccharide-induced thymic atrophy. J Immunol. (2006) 177:169-76. doi: 10.4049/jimmunol.177.1.169

46. Wang SD, Huang KJ, Lin YS, Lei HY. Sepsis-induced apoptosis of the thymocytes in mice. J Immunol. (1994) 152:5014-21.
47. Wyllie H. Glucocorticoid-induced thymocyte apoptosis is associated with endogenous endonuclease activation. Nature. (1980) 284:555-6. doi: 10.1038/284555a0

48. Yuuki H, Yoshikai Y, Kishihara K, Matsuzaki G, Ayukawa K, Nomoto K. The expression and sequences of $\mathrm{T}$ cell antigen receptor beta-chain genes in the thymus at an early stage after sublethal irradiation. J Immunol. (1989) 142:3683-91.

49. Zúñiga-Pflücker JC, Jiang D, Schwartzberg PL, Lenardo MJ. Sublethal gamma-radiation induces differentiation of CD4-/CD8- into $\mathrm{CD} 4+/ \mathrm{CD} 8+$ thymocytes without $\mathrm{T}$ cell receptor beta rearrangement in recombinase activation gene 2-/- mice. J Exp Med. (1994) 180:1517-21. doi: 10.1084/jem.180.4.1517

50. Ivanov VN, Nikolić-Zugić J. Biochemical and kinetic characterization of the glucocorticoid-induced apoptosis of immature CD4+CD8+ thymocytes. Int Immunol. (1998) 10:1807-7. doi: 10.1093/intimm/10.12.1807

51. Goldberg GL, King CG, Nejat RA, Suh DY, Smith OM, Bretz JC, et al. Luteinizing hormone-releasing hormone enhances $\mathrm{T}$ cell recovery following allogeneic bone marrow transplantation. J Immunol. (2009) 182:5846-54. doi: 10.4049/jimmunol.0801458

52. Heng TS, Goldberg GL, Gray DH, Sutherland JS, Chidgey AP, Boyd RL. Effects of castration on thymocyte development in two different models of thymic involution. J Immunol. (2005) 175:2982-93. doi: 10.4049/jimmunol.175.5.2982

53. Sutherland JS, Goldberg GL, Hammett MV, Uldrich AP, Berzins SP, Heng TS, et al. Activation of thymic regeneration in mice and humans following androgen blockade. J Immunol. (2005) 175:2741-53. doi: 10.4049/jimmunol.175.4.2741

54. Lai KP, Lai JJ, Chang P, Altuwaijri S, Hsu JW, Chuang KH, et al. Targeting thymic epithelia AR enhances T-cell reconstitution and bone marrow transplant grafting efficacy. Mol Endocrinol. (2013) 27:25-37. doi: 10.1210/me.2012-1244

55. Zoller AL, Kersh GJ. Estrogen induces thymic atrophy by eliminating early thymic progenitors and inhibiting proliferation of beta-selected thymocytes. $J$ Immunol. (2006) 176:7371-8. doi: 10.4049/jimmunol.176.12.7371

56. Jaffe HL. The influence of the suprarenal gland on the thymus: I. regeneration of the thymus following double suprarenalectomy in the rat. J Exp Med. (1924) 40:325-42. doi: 10.1084/jem.40.3.325

57. Miller JF. Immunological function of the thymus. Lancet. (1961) 2:748-9. doi: 10.1016/S0140-6736(61)90693-6

58. Kaneko KB, Tateishi R, Miyao T, Takakura Y, Akiyama N, Yokota R, et al. Quantitative analysis reveals reciprocal regulations underlying recovery dynamics of thymocytes and thymic environment in mice. Commun Biol. (2019) 2:444. doi: 10.1038/s42003-019-0688-8

59. Dudakov JA, Hanash AM, Jenq RR, Young LF, Ghosh A, Singer NV, et al. Interleukin-22 drives endogenous thymic regeneration in mice. Science. (2012) 336:91-5. doi: 10.1126/science.1218004

60. Dumont-Lagacé M, Gerbe H, Daouda T, Laverdure JP, Brochu S, Lemieux $\mathrm{S}$, et al. Detection of quiescent radioresistant epithelial progenitors in the adult thymus. Front Immunol. (2017) 8:1717. doi: 10.3389/fimmu.2017. 01717

61. Lepletier A, Hun ML, Hammett MV, Wong K, Naeem H, Hedger M, et al. Interplay between Follistatin, Activin A, and BMP4 signaling regulates postnatal thymic epithelial progenitor cell differentiation during aging. Cell Rep. (2019) 27:3887-901.e4. doi: 10.1016/j.celrep.2019. 05.045

62. Pan B, Liu J, Zhang Y, Sun Y, Wu Q, Zhao K, et al. Acute ablation of DP thymocytes induces up-regulation of IL-22 and Foxn1 in TECs. Clin Immunol. (2014) 150:101-8. doi: 10.1016/j.clim.2013.11.002

63. Pan B, Wang D, Li L, Shang L, Xia F, Zhang F, et al. IL-22 accelerates thymus regeneration via Stat3/Mcl-1 and decreases chronic graft-versus-host disease in mice after allotransplants. Biol Blood Marrow Transplant. (2019) 25:1911-19. doi: 10.1016/j.bbmt.2019.06.002

64. Rossi S, Blazar BR, Farrell CL, Danilenko DM, Lacey DL, Weinberg KI, et al. Keratinocyte growth factor preserves normal thymopoiesis and thymic microenvironment during experimental graft-versus-host disease. Blood. (2002) 100:682-91. doi: 10.1182/blood.V100.2.682 
65. Min D, Taylor PA, Panoskaltsis-Mortari A, Chung B, Danilenko DM, Farrell C, et al. Protection from thymic epithelial cell injury by keratinocyte growth factor: a new approach to improve thymic and peripheral T-cell reconstitution after bone marrow transplantation. Blood. (2002) 99:4592-600. doi: 10.1182/blood.V99.12.4592

66. Alpdogan O, Hubbard VM, Smith OM, Patel N, Lu S, Goldberg GL, et al. Keratinocyte growth factor (KGF) is required for postnatal thymic regeneration. Blood. (2006) 107:2453-60. doi: 10.1182/blood-2005-07-2831

67. Rossi SW, Jeker LT, Ueno T, Kuse S, Keller MP, Zuklys S, et al. Keratinocyte growth factor (KGF) enhances postnatal T-cell development via enhancements in proliferation and function of thymic epithelial cells. Blood. (2007) 109:3803-11. doi: 10.1182/blood-2006-10-0 49767

68. Wertheimer T, Velardi E, Tsai J, Cooper K, Xiao S, Kloss CC, et al. Production of BMP4 by endothelial cells is crucial for endogenous thymic regeneration. Sci Immunol. (2018) 3:eaal2736. doi: 10.1126/sciimmunol.aa 12736
69. Blanpain C, Fuchs E. Stem cell plasticity. Plasticity of epithelial stem cells in tissue regeneration. Science. (2014) 344:1242281. doi: $10.1126 /$ science. 1242281

70. Snippert HJ, Clevers H. Tracking adult stem cells. EMBO Rep. (2011) 12:11322. doi: 10.1038/embor.2010.216

Conflict of Interest: The authors declare that the research was conducted in the absence of any commercial or financial relationships that could be construed as a potential conflict of interest.

Copyright (c) 2021 Ishikawa, Akiyama and Akiyama. This is an open-access article distributed under the terms of the Creative Commons Attribution License (CC BY). The use, distribution or reproduction in other forums is permitted, provided the original author(s) and the copyright owner(s) are credited and that the original publication in this journal is cited, in accordance with accepted academic practice. No use, distribution or reproduction is permitted which does not comply with these terms. 